\title{
Factores de riesgo para sobrepeso $y$ obesidad en lactantes
}

\author{
Risk factors for overweight \\ and obesity in infants
}

\begin{abstract}
Introduction: At one year of age, factors that favor the development of obesity and other metabolic disorders in later stages of life are present. Objective: To identify perinatal, societal, and dietary risk factors for excess weight and obesity in 12 months infants. Subjects and methods: Case-control studies with infants under the care of Family Medicine Units. These cases studied were overweight or obese children, and control cases were children at a healthy weight. Each group included 152 infants, who were evaluated based on their responses to a questionnaire, their records, and perinatal, societal, and dietary variables. Results: The variables with statistical significance were high pre-gestational weight, high gestational weight gain (OR 1.79-3.88), absence of breastfeeding and exclusive formula feeding (OR 2.02 and 1.75). Conclusion: In the presence of pre-pregnancy obesity and exclusive formula feeding, there is a $64.4 \%$ greater risk of becoming overweight or obese at 12 months old.

Key words: infants, excess weight, weight problems, obesity, perinatal, diet.
\end{abstract}

Laura Cu F. (1-2)

Enrique Villarreal R. (1)

Beatríz Rangel P. (2)

Liliana Galicia R. (1)

Emma Vargas D. (1)

Lidia Martínez G. (1)

(1) Unidad de Investigación Epidemiológica y de Servicios de Salud Querétaro, Instituto Mexicano del Seguro Social. Querétaro, México. (2) Facultad de Ciencias Naturales, Universidad Autónoma de Querétaro; Querétaro, México.

Dirigir la correspondencia a: Profesora Laura Cu Flores Unidad de Investigación Epidemiológica y de Servicios de Salud Querétaro. Instituto Mexicano del Seguro Social. Querétaro, México.

Teléfono: 011524421991228 E-mail: baly2105@hotmail.com

Este trabajo fue recibido el 25 de Septiembre de 2014 y aceptado para ser publicado el 25 de Marzo de 2015.

\section{INTRODUCCIÓN}

Durante el primer año de vida se produce la etapa de crecimiento y desarrollo postnatal más acelerada del ser humano (1). La nutrición de los niños en los primeros meses es importante porque o limita o favorece el potencial de crecimiento físico y el desarrollo intelectual en etapas posteriores; determina consecuencias metabólicas importantes durante la infancia tardía y la adolescencia que pueden influir en la calidad de vida en la adultez (2). Se ha demostrado que la mayor incidencia de morbilidad y mortalidad relacionada con la nutrición se presenta durante el primer año de vida del ser humano (3).

El estado nutricional en esta etapa es el mejor indicador de salud. La evaluación de los indicadores de crecimiento permite determinar si un niño crece normalmente o presenta problemas, ya sea por estados carenciales o por exceso (sobrepeso y obesidad) (4), que requirieran de toma de decisiones y acciones para mejorar su condición (5).

En México, la obesidad es un problema de salud pública que aqueja a la población pediátrica y que impacta el panorama epidemiológico del país (3). La obesidad se define como un exceso de grasa corporal debido a un desequilibrio entre el ingreso y el gasto energético del organismo (5).

La obesidad infantil debe abordarse desde una perspectiva multicausal6, en la que tanto el individuo como los padres, el medio familiar o el ambiente en general, pueden favorecen su aparición (7). Desde el periodo gestacional y pregestacional, factores como la obesidad materna, el incremento de peso gestacional de la madre, la multiparidad, el peso del producto al nacimiento y la prematurez, son determinantes de una mayor susceptibilidad (8).

El ambiente obesogénico en el que se desarrolla el individuo (9) bajo la influencia de los hábitos y conductas alimentarias de los padres y hermanos, la presencia de obesidad en el núcleo familiar (10), el cuidado de los lactantes dentro o fuera de casa, la preparación académica de los padres y el nivel socioeconómico, forman parte del entorno social que acompaña a la obesidad (11).

La alimentación en los primeros meses de vida del lactante, ya sea con base en fórmulas lácteas o al seno materno, en conjunto con el tiempo de duración de éstas, la edad y características del inicio de la alimentación complementaria, el consumo de alimentos con alta densidad energética (12), resulta por sí sola o en asociación con los factores antes mencionados, en un detonante para la aparición de obesidad (13) en edades tempranas, lo que impacta la morbilidad y mortalidad a corto, mediano y largo plazo (14).

El presente estudio consideró importante identificar los factores de riesgo perinatales, sociales y alimentarios que favorecen el desarrollo de sobrepeso y obesidad en lactantes de un año de edad. 


\section{SUJETOS Y MÉTODOS}

Se realizó un estudio de casos y controles en niñas y niños lactantes de un año de edad que acudieron a consulta externa de medicina familiar o con la enfermera materno-infantil, en clínicas de primer nivel de atención del (IMSS) del Instituto Mexicano del Seguro Social, Delegación Querétaro, correspondientes a las Unidades de Medicina Familiar números 5,9,13,15 y 16, en el período de enero de 2013 a diciembre de 2013.

Los grupos de estudio fueron aquellos con estado nutricional adecuado (control) y los que presentaron sobrepeso y obesidad (casos).

Se incluyeron pacientes que contaran con un peso adecuado al nacimiento (entre 2,500g y 4,000g en un recién nacido a término), padre o tutor del lactante que aceptara participar en el estudio y lactantes con padre o madre asegurados. Se excluyeron lactantes con diagnóstico de reflujo gastroesofágico o con alguno de los siguientes antecedentes; prematurez (menos de 37 semanas de gestación), desnutrición o con enfermedades metabólicas, infecciosas, inmunológicas u oncológicas. Se eliminaron las entrevistas incompletas.

El tamaño de la muestra ( $n=152$ por grupo) se calculó con la fórmula para casos y controles pareado, con una confianza del 95\% (Zalfa= 1.96), poder de la prueba $80 \%$ (Zbeta=0.84), con (OR) 2, odds ratio mínima que se espera encontrar y porcentaje de discordancia de 0.462, Pdiscordante $=0.462$, asumiendo que el porcentaje de discordancia entre los casos y los controles se calcula a partir de $(b+c) / N$, es decir, la suma del total de controles expuestos (63) más el total de casos no expuestos (78), dividido entre el total de casos y controles (304).

La técnica muestral fue estratificada por conglomerados (considerando como conglomerado a cada una de las Unidades de Medicina Familiar; 5,9,13,15,16). El muestreo fue por cuota, durante los meses asignados para la recolección de la muestra, los pacientes fueron captados en la sala de espera de los servicios mencionados, durante los turnos matutino y vespertino.

Contando con la autorización del comité de ética, y con la firma de consentimiento informado por el padre o tutor para participar en el estudio, se aplicó un cuestionario para recolectar la información de las variables de estudio. La captura se clasificó en 3 grupos, con sus respectivas variables cada uno; se corroboró la información del cuestionario en expedientes médicos.

\section{Variables perinatales}

Obesidad materna pregestacional

Incremento de peso gestacional

Multiparidad

\section{Variables sociales}

Obesidad del padre

Asistencia a guarderías

Escolaridad materna

\section{Variables alimentarias}

Lactancia materna

Alimentación con fórmula láctea

Alimentación mixta

\section{Alimentación complementaria}

Ingesta de bebidas endulzadas (jugos)

Edad de inicio de consumo de bebidas endulzadas (jugos)

Frecuencia en el consumo de bebidas endulzadas (jugos)
Índice de masa corporal (IMC) de la madre previo a la gestación, criterio de corte conforme a la NOM-008-SSA3-2010 (23).

Peso en kilos ganado durante gestación, relacionados al IMC pregestacional, conforme a criterios del Institute of Medicine (IOM) 2009 (24).

Número total de embarazos (3 o más), al momento de la entrevista

Establecido por el IMC de acuerdo a la NOM-008-SSA3-2010 (23).

Asistencia del lactante a guarderías 4 meses o más en el primer año de vida.

Último grado de estudios de la madre, definida como baja cuando fue de primaria o menor.

Alimentación del lactante únicamente con leche materna. Se definió como exclusiva cuando la lactancia materna se presentó de esta manera: por 4 meses o más.

Alimentación del lactante con fórmulas lácteas. Se definió como exclusiva cuando la alimentación del lactante constó únicamente en fórmulas lácteas.

Alimentación del lactante combinando fórmula láctea y leche materna (3 meses como mínimo)

El lactante consumió alimentos diferentes a la leche materna y fórmulas lácteas. Definida como temprana, antes de los 4 meses de edad de acuerdo a la FAO.

El lactante ha consumido desde el nacimiento a la fecha bebidas endulzadas (jugos).

Edad del lactante en la que se inició la ingesta de jugos. Definiéndose como temprana, antes de los 4 meses.

Frecuencia de consumo semanal de jugos, desde que inició con la ingesta de los mismos. Definido como consumo frecuente, más de 3 veces/semana. 
La evaluación del estado nutricional se realizó mediente la medición del peso y longitud. El peso se tomó verificando que el lactante portara pañal seco; se utilizó una báscula digital infantil previamente calibrada, marca Seca 334 con precisión de 50 g y la longitud se tomó con un infantómetro marca Seca 334. El diagnóstico se realizó con el indicador: peso para la longitud para niños y niñas del nacimiento a los 2 años. Se utilizaron las tablas de puntaje Z, de la OMS, 2006 (20). Los criterios de corte fueron:

\begin{tabular}{|c|c|}
\hline Peso normal & $\begin{array}{l}\text { A los que se encontraron por arriba de la } \\
\text { mediana hasta la línea de puntuación Z+2 } \\
\text { y por debajo de la mediana hasta la línea } \\
\text { de puntuación Z- } 2 \text {. }\end{array}$ \\
\hline
\end{tabular}

Sobrepeso

Por arriba de la línea de puntuación $Z+2$ y por debajo de $\mathrm{Z}+3$.

Obesidad

Por arriba de la línea de puntuación Z+3.

Se realizó estadistica descriptiva: porcentajes, promedios, desviación estándar e intervalos de confianza del 95\%. El análisis inferencial contempló la prueba de $\mathrm{Chi}^{2}$, OR, regresión logística múltiple y se aplicó el modelo de probabilidad de riesgo.

Los modelos multivariados diseñados constaron de la asociación de diferentes variables con diversos intentos de agrupación, se eligió el modelo que presentó la mejor significancia estadística. Sin embargo, se observó colinealidad en las variables que lo integraban, por lo que al encontrarse tal asociación, se replantearon diversos intentos por formar un modelo que pudiera explicar el sobrepeso y la obesidad, libre de colinealidad y que se caracterizara por tener la mejor significancia estadística entre los modelos formados. Una vez obtenido el modelo se propuso un análisis de probabilidad de riesgo.

\section{RESULTADOS}

En la tabla 1 se observa que existió una mayor proporción de niñas con sobrepeso y obesidad en comparación con los niños; incremento de peso de las madres durante la gestación expresado en kilos fue mayor en el grupo de lactantes con sobrepeso y obesidad, en aproximadamente 1.89 kilos.

Al realizar el análisis con $\mathrm{Chi}^{2}$, se observó que de las variables perinatales la obesidad pregestacional y el incremento de peso gestacional elevado mostraron significancia estadística con una OR 1,79 y 3,88 respectivamente. En las variables alimentarias se presentó un valor de p significativo; la ausencia de lactancia materna OR 2,02 y la alimentación con fórmula láctea OR 1,75 Las variables restantes no mostraron significancia estadística (tabla 2).

El modelo de regresión logística múltiple que mejor explicó la asociación entre las variables, fue el integrado por el incremento de peso gestacional y la alimentación con fórmula láctea, con un valor de $\mathrm{p}=0.002$ (tabla 3).

El análisis de probabilidad de riesgo, tomando el modelo con las variables de incremento de peso gestacional y la alimentación con fórmula láctea, presentó una probabilidad de riesgo 64\% mayor de desarrollar sobrepeso y obesidad al año de edad cuando está presente la obesidad materna pregestacional y el lactante es alimentado con fórmula láctea (tabla 4).

\section{DISCUSIÓN}

$\mathrm{Al}$ analizar las variables perinatales; la obesidad pregestacional y el incremento de peso gestacional se comportaron como factores de riesgo, ambas circunstancias concuerdan con resultados referidos en la literatura (18), en los que se establece que el IMC materno al comienzo del embarazo y el incremento de peso durante la gestación son un factor predictivo altamente asociado al peso de nacimiento (22) y al IMC de los lactantes desde etapas muy tempranas, involucrando procesos de adaptación metabólica y hormonal que persisten a lo largo de la vida $(9,15)$.

En México, es preocupante la alta prevalencia de obesidad en la población en edad fértil (19) y ante la evidente falta de planeación de los embarazos, se presenta un panorama que impacta en el riesgo de sobrepeso y obesidad en los lactantes. Se observa que el incremento de peso durante la gestación es mayor en mujeres que presentaban con obesidad pregesta-

\section{TABLA 1}

Características de la población de estudio.

\begin{tabular}{|c|c|c|}
\hline & \multicolumn{2}{|c|}{ Diagnóstico Nutricional } \\
\hline & $\begin{array}{l}\text { Adecuado } \\
\text { N (\%) }\end{array}$ & $\begin{array}{c}\text { Sobrepeso y obesidad } \\
\text { N (\%) }\end{array}$ \\
\hline \multicolumn{3}{|l|}{ Sexo de los lactante } \\
\hline Masculino & $79(52,00 \%)$ & $70(46,00 \%)$ \\
\hline Femenino & $73(48,00 \%)$ & $82(54,00 \%)$ \\
\hline Edad materna & $26,36+5,64^{(a)}$ & $27,22+6,29$ \\
\hline Gestas & $1,98+1,04$ & $1,90+1,07$ \\
\hline IMC pregestacional & $25,04+4,14$ & $25,45+3,49$ \\
\hline $\begin{array}{l}\text { Peso }(\mathrm{Kg}) \text { ganado } \\
\text { durante la gestación }\end{array}$ & $10,70+4,88$ & $12,59+4,84$ \\
\hline $\begin{array}{l}\text { ara cada uno de los gru } \\
\text { atos de se expresan en }+ \\
\text { edia }+D E\end{array}$ & rcentaje. & \\
\hline
\end{tabular}


cional $(21,23)$, situación que en muchas ocasiones dentro la atención prenatal, no contempla acciones contundentes para minimizar dicho riesgo.

De los factores alimentarios; la ausencia de lactancia materna y la alimentación con fórmulas lácteas, presentaron riesgo para el desarrollo de obesidad. Es bien conocido el factor protector que tiene la lactancia materna en prevenir el desarrollo de la obesidad $(16,21)$, pero es innegable la inmensa cantidad de situaciones que limitan esta práctica, particularmente el rol actual de la mujer en actividades laborales y la falta de sensibilización y de conocimiento que se tiene sobre los beneficios de la lactancia (25). Pese a los grandes esfuerzos por parte de los servicios de salud y políticas gubernamentales, aún no se ha logrado una cultura que favorezca la lactancia materna, que logre que las mujeres inicien esta práctica tempranamente y se continúe con éxito durante 6 meses. La Encuesta Nacional de Nutrición y Salud de 2012 refleja que en los lactantes menores de 6 meses, la práctica de lactancia materna exclusiva mostró un notable deterioro al pasar de $22.3 \%$ en el año 2006 a 14.5\%. Este descenso explica el incremento en el porcentaje de niños que consumen fórmula lactea (27).

Es innegable que la implementación de la lactancia materna involucra muchas aspectos; en el ámbito laboral, ambientes de trabajo que favorezcan la lactancia materna, la reducción de jornadas en los períodos de lactancia, prolongación del período de incapacidad materna sin arriesgar la estabilidad laboral, guarderías en centros de trabajo e instalaciones especiales para que las madres lacten; son algunas condiciones con que cuentan otros países y que limitan el consumo de fórmulas

TABLA 2

Factores de riesgo asociados a sobrepeso y obesidad.

\begin{tabular}{|c|c|c|c|c|c|c|}
\hline \multirow[t]{2}{*}{ Factores } & \multirow{3}{*}{$\begin{array}{c}\text { Sobrepeso y } \\
\text { obesidad } \\
\%\end{array}$} & \multirow{3}{*}{$\begin{array}{c}\text { Adecuado } \\
\%\end{array}$} & \multirow[t]{3}{*}{ OR } & \multicolumn{2}{|c|}{ IC $95 \%$} & \multirow[t]{2}{*}{$\mathrm{p}$} \\
\hline & & & & Inferior & Superior & \\
\hline Perinatales & & & & & & \\
\hline Obesidad pregestacional & 57,20 & 42,80 & 1,79 & 1,13 & 2,82 & 0,01 \\
\hline Incremento de peso gestacional elevado & 60,50 & 28,30 & 3,88 & 2,40 & 6,28 & 0,00 \\
\hline Multiparidad & 9,20 & 7,90 & 1,18 & 0,52 & 2,65 & 0,68 \\
\hline \multicolumn{7}{|l|}{ Sociales } \\
\hline Obesidad del padre & 49,30 & 50,70 & 0,94 & 0,54 & 1,69 & 0,81 \\
\hline Asistencia a guarderías & 19,10 & 19,70 & 0,05 & 0,38 & 1,46 & 0,88 \\
\hline Escolaridad baja de la madre & 38,20 & 44,10 & 0,78 & 0,49 & 1,23 & 0,29 \\
\hline \multicolumn{7}{|l|}{ Alimentarios } \\
\hline Ausencia de lactancia materna exclusiva & 7,70 & 60,50 & 2,02 & 1,23 & 3,31 & 0,00 \\
\hline Alimentación con fórmula exclusiva & 52,00 & 38,20 & 1,75 & 1,11 & 2,76 & 0,01 \\
\hline Alimentación mixta & 35,50 & 38,20 & 0,89 & 0,56 & 1,42 & 0,63 \\
\hline Alimentación complementaria temprana & 26,30 & 22,40 & 1,23 & 0,73 & 2,09 & 0,42 \\
\hline Ingesta de jugos & 75,70 & 69,70 & 1,34 & 0,81 & 2,23 & 0,24 \\
\hline Inicio temprano en la ingesta de jugos & 27,60 & 19,70 & 1,55 & 0,91 & 2,65 & 0,10 \\
\hline Frecuencia en el consumo de jugos & 44,70 & 34,90 & 0,51 & 0,95 & 2,40 & 0,07 \\
\hline
\end{tabular}

TABLA 3

Modelo de regresión logística múltiple en los grupos casos y control.

\begin{tabular}{|c|c|c|c|c|}
\hline \multirow[b]{2}{*}{ Variable } & \multicolumn{2}{|c|}{$\begin{array}{l}\mathrm{Chi}^{2} \\
1,99\end{array}$} & \multicolumn{2}{|c|}{$\begin{array}{c}p \\
0,00\end{array}$} \\
\hline & Betas & Estadístico & OR & $\mathrm{p}$ \\
\hline Obesidad pregestacional & 0,57 & 6,05 & 1,77 & 0,01 \\
\hline Alimentación con fórmula láctea exclusiva & 0,55 & 5,54 & 1,74 & 0,01 \\
\hline
\end{tabular}


lácteas $(17,21,25,26)$.

En el análisis grupal de las variables en que la obesidad pregestacional y el incremento de peso gestacional presentaron colinealidad, se presume que tal asociación es consecuencia de malos hábitos de alimentación. La obesidad presente desde etapas previas al embarazo demostró una estrecha relación con la elevada ingesta alimentaria a lo largo de la gestación, resultando en un incremento de peso gestacional mayor al esperado, condición que ya ha sido referido en otras revisiones $(9,13,17)$, y que en el contexto de la cultura en México, esta obesidad gestacional es socialmente aceptada. La colinealidad entre estas variables, precisó la eliminación de la variable incremento de peso gestacional, en la integración de los modelos. El modelo que mejor explicó el sobrepeso y la obesidad en los lactantes de 12 meses fue el que asoció la obesidad pregestacional y la alimentación con fórmula láctea.

Al plantear un modelo de probabilidad de riesgo cuando existe obesidad pregestacional y alimentación con fórmula láctea exclusiva, la probabilidad de desarrollar sobrepeso y obesidad a los 12 meses de edad fue 64.38\%. Un aspecto muy interesante en este modelo, es que la presencia de una condición previa a la gestación (obesidad pregestacional) está favoreciendo la obesidad en los lactantes antes de ser concebidos y que tanto esta como la alimentación con fórmula láctea exclusiva son finalmente resultado de malas prácticas de alimentación, orientación y de trabajo preventivo a lo largo del embarazo, lo que se ve reflejado en la morbilidad y mortalidad materna e infantil $(9,14)$.

Como anteriormente se planteó, hay un pobre conocimiento en relación a la alimentación, lo que impide realizar elecciones adecuadas sobre la forma más saludable de alimentarse en cada etapa de la vida $(11,14,23)$ y la importancia de adoptar prácticas como la lactancia materna (25). Se sabe que el período de gestación es la etapa determinante en que la mujer decidirá si realizará o no lactancia materna y por cuánto tiempo la llevará a cabo; lo que establece que el contacto desde etapas tempranas de gestación, el adiestramiento en técnicas de amamantamiento y la sensibilización en primer nivel de atención son determinante para la elección de esta práctica (26).

Otras situaciones que condicionan la elección de alimentos, están influenciadas por la disponibilidad de los mismos, su costo, el tiempo de preparación y la mercadotecnia, entre otros. Sin embargo, contar con una sensibilización de la población en materia de nutrición, puede lograr hábitos alimentarios más saludables, dentro de las limitaciones que en forma individual puedan presentarse $(2,14)$

\section{CONCLUSIÓN}

Se concluye que con la presencia de obesidad pregestacional y de alimentación con fórmula láctea exclusiva, existe un riesgo $64.38 \%$ mayor de desarrollar sobrepeso y obesidad a los 12 meses de edad.

\section{RESUMEN}

Introducción: Al año de vida existen factores que favorecen el desarrollo de obesidad y alteraciones metabólicas en etapas posteriores. Objetivo: Identificar factores de riesgo perinatales, sociales y alimentarios de sobrepeso y obesidad en lactantes de 12 meses de edad. Sujetos y método: Estudio de casos y controles en lactantes, que acuden a Unidades de Medicina Familiar, considerando casos; niños con sobrepeso u obesidad y controles: peso adecuado. Se incluyeron 152 lactantes por grupo, a los cuales se les estudió mediante un cuestionario y revisión de expedientes, variables perinatales, sociales y alimentarias. Resultados: Las variables con significancia estadística fueron: peso pre-gestacional de la madre elevado, incremento de peso gestacional elevado; OR 1,79 y 3,88 ausencia de lactancia materna y alimentación con fórmula láctea exclusiva, OR 2,02 y 1,75.Conclusión: en presencia de obesidad pregestacional y alimentación con formula láctea exclusiva, existe un riesgo 64,38\% mayor de desarrollar sobrepeso u obesidad a los 12 meses de edad.

Palabras clave: lactantes; sobrepeso; obesidad; perinatales; alimentarios.

\section{BIBLIOGRAFÍA}

1. Thompson-Chagoyán O. La desnutrición infantil como un problema de salud. Rev Hosp Gral Dr. M Gea González. 2000;3:5-8

2. Castillo-Duran C, Balboa P,Torrejon C, Bascuñan K, uauy R. Alimentación normal en el niño menor de 2 años. Rev Chil Pediatr. 2013;84(5):565-72.

3. Pérez E, Morales M, Grajales I. Panorama epidemiológico de la obesidad en México. Rev Mex Enf Cardiol. 2006;14:62-4.

4. Bueno M. Endocrinología pediátrica en el siglo XXI. EI modelo de la obesidad pasado presente y futuro. Rev Esp Nutr Comunitaria. 2004; 10:192-6.

5. Durá Travé E, Gallinas Victorinao F. Evolución natural del exceso de peso corporal (sobrepeso y obesidad). An Pediatr (Barc). 2013;79(5)300-16.

6. Reilly J, Armstronh J, Dorosty A, Emmett P, Colin-Steer R, et al. Early life risk factors for obesity in childhood: cohort study. BMJ; 331(7514):453-4.

7. Cano Garcinuño A, Alberola López S, Casares Alonso I,

\section{TABLA 4}

Cálculo de la probabilidad de riesgo para sobrepeso y obesidad.

Obesidad

pregestacional
Alimentación con

fórmula láctea exclusiva
Probabilidad de

desarrollar obesidad $\%$

64,38

51,95

50,40

37,86 
Pérez García. Desigualidades sociales en la prevalencia de sobrepeso y obesidad en adolescentes. An Pediatr (Barc).2010;73:241-58

8. Anzman B. Parental influence on children's early eating environments and obesity risk: implications for prevention Birch Internat J Obes. 2011;34:1116-24.

9. Garibay-Nieto N. Impacto de la programación fetal y la nutrición durante el primer año de vida en el desarrollo de obesidad y sus complicaciones. Bol Med Hosp Infant Mex. 2008;65:451-67.

10. Agostoni C, Decsi T, Fewtrell M, Goulet O, Kolacet S. et al. Complementary Feeding: A Commentary by the Espghan Committee on Nutrition. J Pediatr Gastroenterol Nutr.2008;46:99-110.

11. Bracho M, Ramos H. Percepción materna del estado nutricional de sus hijos: ¿es un factor de riesgo para presentar malnutrición por exceso? Rev Chil Pediatr. 2007;78:20-7.

12. Gokkay C, Turan J, Portalci A, Neyzi O. Growth infants during the first year of life according to feeding regimen in the first 4 months. J Trop Pediatr. 2003;49:6-12.

13. Jiménez $R$, Curbelo J, Peñalver R. Relación del tipo de alimentación con algunas variables de crecimiento, estado nutricional y morbilidad del lactante. Colomb Med.2005;36:19-25.

14. Díaz-Argüelles $V$, Ramírez-Corría. La alimentación inadecuada del lactante sano y sus consecuencias. Rev Cubana Pediatr. 2005;77:113-9.

15. Jiménez $R$, Curbelo J, Peñalver R. Relación del tipo de alimentación con algunas variables de crecimiento, estado nutricional y morbilidad del lactante. Colomb Med. 2005;36:19-25.

16. Kramer S, Chalmers B, Hodnett E, Hodnett $E$, Sevkovskaya Z, Dzikovich I, et al. Promotion of Breastfeeding Intervention Trial (PROBIT) JAMA. 2001;285:413-20.

17. Loaíza S, Bustos P. Factores asociados al exceso de peso durante el primer año de vida. Rev Chil Pediatr. 2007;78:143-50.
18. Macías A, Hernández M, Ariosa J, Alegret M. Crecimiento prenatal y crecimiento posnatal asociados a obesidad en escolares. Rev Cubana Invest Biomed. 2007;26:1:16.

19. Gutierrez JP, Rivera-Dommarco J, Shamah-Levy T, Villalpando-Hernández S, Franco A, Cuevas-Nasu et. al. Encuesta Nacional de Salud y Nutricion 2012. Resultados Nacionales.Cuernavaca, México: Instituto Nacional de Salud Pública $(M X), 2012$.

20. Onís M, Onyango W, Borghi E, Siyam A, Nacida C, Siekmann J. et al. Bulletin of the World Health Organization. 2007;85:660-7.

21. Marieke $H$, Manon $E$, Karien S. Overweight at age two years in a multi-ethnic cohort ( $A B C D$ study): the role of prenatal factors, birth outcomes and postnatal factors $B M C$ Public Health. 2011;11:611-9.

22. Lagos L, Espinoza R, Orellana J. Estado nutritivo materno inicial y peso promedio de sus recién nacidos a término. Rev Chil Nutr. 2004;31(1):52-7.

23. Herring S,Oken E. Ganancia de peso durante el embarazo: Su importancia para el estado de salud materno-infantil. Ann Nestlé [Esp]. 2010;68:17-8.

24. Norma oficial mexicana, NOM-008-SSA3-2010. Para el tratamiento integral del sobrepeso y la obesidad. Disponible en: http://www.dof.gob. $m x /$ nota_detalle.php?codigo $=51$ 54226\&fecha=04/08/2010 [Consultada: 04/04/2014].

25. Navarro-Estrella M, Duque-López M, Trejo y Pérez J. Factores que influyen en el abandono temprano de la lactancia por mujeres trabajadoras. Salud Publica Mex. 2003;45:276-84.

26. Felix-Vlaenzuela J, Martínez-Caldera E, Sotelo-Ham Elma. Prevalencia y factores biosociales asociados al abandono de la lactancia materna exclusiva. Pediatr Mex. 2011;13 (2):47-56.

27. González de Cosio T, Escobar-Zaragoza L, González-Castell L, Rivera-Dommarco M.Prácticas de alimentación infantily deterioro de la lactancia materna en México. Salud Pública Méx. 2013; 55(2):170-9 